\title{
Reimagining GIS Instruction through Concept-Based Learning
}

\author{
Sara Lafia ${ }^{\mathrm{a}}$, Rui Zhu ${ }^{\mathrm{b}}$, Blake Regalia ${ }^{\mathrm{b}}$, and Werner Kuhn ${ }^{\mathrm{b}}$ \\ a ICPSR, University of Michigan, Ann Arbor, USA \\ ${ }^{b}$ Department of Geography, UC Santa Barbara, Santa Barbara, USA
}

Correspondence: slafia@umich.edu

\begin{abstract}
Research in geographic information science has not yet found clear answers to the questions of what geographic information is about or what a geographic information system (GIS) contains. This lack of consensus makes it especially challenging to teach and learn GIS. Existing pedagogical approaches either focus on the representational level of data (e.g., "raster and vector") or are too generic (e.g., "geo-referenced information"). This characterization of GIS and its content is difficult for learners to transfer and apply broadly. As instructors, we approach the challenge of teaching GIS from a conceptual basis. We describe our process to develop a set of core concepts of spatial information, which we use to redesign an undergraduate-level introductory GIS course. Our intervention focuses instruction on the kinds of questions that geographic information enables before training students to produce workflows and answers through system commands. The course redesign complements and informs ongoing research on core concepts of spatial information. Our results demonstrate that GIS courses can deliver more than software training, indicating both theoretical gains and didactic challenges.
\end{abstract}

Keywords. GIS education and training, geographic information systems, core concepts of spatial information, interdisciplinary research, concept based learning

\section{Introduction}

A quarter century into labeling academic studies around GIS (geographic information systems) as science (Goodchild, 1992), we still lack a consensus on what this science is about or, technically speaking, what GIScience studies and what constitutes geographic information. Clearly geographic information is about more than "raster and vector data " or "geo-referenced information"- but what exactly is it? This lack of consensus is particularly apparent in the context of GIS instruction. A related consequence is the difficulty of explaining to an economist or biologist, for example, what GIS can do for them.

The ambiguous definition of GIS as both a tool and a scientific endeavor shapes core concepts and learning outcomes (Wright et al., 1997). As a tool, GIS plays a supporting role in applied problem solving for other research endeavors; for example, it is an enabling technology for the study of botany, history, and many other subjects (Kerski et al., 2013). In a tool-centric view of GIS, the core concepts that students must acquire involve learning the software commands that one performs with a GIS such as data "capture", "manipulation", and "integration" (Raper and Green, 1992). However, GIScience offers more than a set of tools for interacting with the world (Goodchild, 2006). Learners are increasingly drawn to interdisciplinary GIS methods for answering complex questions across the social, natural, and physical sciences (Kidman and Palmer, 2006; Rickles et al., 2017).

While there is consensus that as a tool, GIS can be used to capture, store, check, and display data related to positions on Earth's surface, it is still unclear what these data describe conceptually; the answer could be "almost anything" including populations, bus schedules, or climate models. A theory of geographic information is needed to bring order to this variety. How can this be done without restricting GIS to some application domains (e.g., terrains or utilities), while still saying something meaningful about the content of data? Is there a level above data models ("raster and vector") or applications ("viewshed analysis"), and below the generic and obvious ("geo-referenced information") at which we can talk about and teach GIS? 
We explore this question by designing an introductory GIS course structured around a set of core concepts of spatial information following those proposed by Kuhn (2012). The goal of the course was to define an appropriate conceptual level at which the contents of a GIS could be meaningfully studied and discussed with first time learners. At this level, all possible GIS contents can be distilled into instances of a small set of core concepts. The development of the course accompanied research to test the core concepts in real-world GIS applications, which involved first developing formal specifications and a high level language around them, and then implementing them in the Python programming language (Kuhn and Ballatore, 2015). The course described in this paper simultaneously informed, and was informed by, this GIScience research.

In the remainder of this paper, we review prior efforts to organize GIS contents and then contrast these with our approach to redesign a GIS course around core concepts. We discuss the connection between teaching and research demonstrated by the hands-on activities that accompanied the course and conclude with open research questions for GIS pedagogy stimulated by the experience of designing the course.

\section{What does a GIS contain?}

The question of what a GIS contains has not yet been explicitly answered at a level above technicalities and below generalities; one could try to infer answers from the organization of GIS courses, academic textbooks, or efforts in the GIScience community to structure knowledge about GIS. These efforts have been largely designed to inventory knowledge and skills gathered from leading scholars and professionals in the field rather than develop comprehensive theories of geographic information; thus, they do not explain what distinguishes geographic information (GI) from other types of information and how GI can be organized in its own right.

The NCGIA Core Curriculum (Kemp and Goodchild, 1991) and the UCGIS Body of Knowledge (GIS\&T BoK) (DeMers, 2009) are the main efforts to organize and define a GIS curriculum. Rather than defining what GIS contain, they take stock of the concepts needed to understand and apply what GIS do, ranging from mathematical to social aspects. The Core Curriculum and GIS\&T BoK frameworks also perpetuate the dual identity of GIS as both a tool and a science by blending tool-centric concepts (e.g., "hardware system software", "raster data structure") with conceptual issues (e.g., "spatial objects and relationships") and operations (e.g., "vector data structures and algorithms"). As a consequence, GIS education has not always distinguished domain concepts from software concepts (Kemp et al., 1992). For a contrasting example, the discipline of statistics clearly distinguishes many of its core concepts (e.g., "probabilities", "distributions") from software concepts or operations (e.g., plotting a histogram in SPSS).

Similar remarks can be made about the nature and goals of popular GIS textbooks, which pragmatically organize contents at the data model level (Bolstad, 2005; Longley et al., 2015). On the other hand, theoretical frameworks from academic literature have pursued more ambitious, unifying frameworks including canonical representations for geographic information (Goodchild et al., 2007; Camara et al., 2014; Zhu et al., 2017), multi-level views of that information beyond current system implementations (Couclelis, 2010), and classifications of analysis functions (Albrecht, 1998). While these frameworks provide insights into the nature of geographic information and computing, they still do not tell an undergraduate student or a colleague from another application domain about unifying concepts or what they can do with a GIS.

In the absence of comprehensive theoretical frameworks that explain and organize GIS contents, many instructors organize training around software modules and system-level commands. A recent survey of over 300 university-level GIS courses found that vector analysis, data models, and data acquisition were the most common topics covered (Wikle and Fagin, 2014). Students are typically given step-by-step instructions on how to apply a particular GIS software to a problem that has been fitted to its commands. While the learning outcomes of such courses may satisfy graduates and employers in the short run, the knowledge and skills acquired have a rather short half-life; computing paradigms change continuously. This style of learning also makes it hard for students to transfer their understanding to other products and problems.

Conceptual frameworks have been proposed to address the knowledge transfer challenges that GIS students face. A recent review of research studies about GIS instruction found that constructivist approaches help students develop technical competence through experiential, hands-on projects (Schulze, 2020). Howarth and Sinton (2011) propose a framework that sequences spatial concepts and combines problem-based learning with cognitive load theory to scaffold student learning. Srivastava and Tait (2012) define threshold concepts for GIS instruction to inform course design. Other efforts to support GIS usability have focused on reordering functions, for example common GIS operations in toolboxes (Gao and Goodchild, 2013). These approaches derive concepts from the GIS\&T Body of Knowledge and as such, do not always distinguish representational spatial concepts (e.g., location, distance, hierarchy) from analytical concepts (e.g., extraction by buffer).

Rather than seeking a canonical form of geographic information or reorganizing system-level commands, our approach to instruction defines a high level view of GIS contents, allowing users to specify their application perspectives. We relate information content to 
user questions rather than to the data formats and system commands that dominate the current image of GIS teaching (Vahedi et al., 2016).

\section{Core concepts in GIS}

The core concepts of spatial information (Kuhn, 2012) offer a means of relating questions to the content of spatial information. They provide a high-level vocabulary for spatial thinking and computing, which can be used to ask and answer questions about phenomena in space and time. The concepts are meant to be generic enough to be applicable to geographic, as well as other spaces. They are comprised of a base concept of location, four concepts of information content, and three concepts of information quality, which are metainformation concepts applicable to all content concepts and their combinations (Table 1).

Table 1. Overview of the core concepts of spatial information

\begin{tabular}{lll}
\hline Base concept & Content concepts & Quality concepts \\
\hline Location & Field & Granularity \\
& Object & Accuracy \\
& Network & Provenance \\
& Event & \\
\hline
\end{tabular}

Each core concept comes with "threshold concepts" (Land et al., 2016), which offer learners transformational insights, or ways of seeing, into an application domain. The following is a brief characterization of each concept in view of its use in GIS instruction. Instruction begins with the base concept of location, which allows students to ask "where" questions. The first threshold concept that students encounter is the idea that location is a spatial relation between a figure and a ground (Talmy, 1983). Following this, students are presented with a series of content concepts: field, object, network, and event. The concept of location is foundational to the field concept, as students learn that fields express values at positions over a given domain.

Students learn that core concepts of spatial information are ways of viewing the world. They select, and in some cases interchange, core concepts of spatial information to produce a desired view of a spatial problem; for instance, students learn that geographic phenomena, such as land cover, can be conceptualized and analyzed as a field or as a set of objects (e.g., discrete parcels of land with attributes). The network concept builds on the object concept, answering questions about connectivity. Students conceptualize events using any combination of core concepts as "participants" (e.g., a rainfall-induced traffic event involves a set of participants including cars as objects, a road network along which they can be located, and a field of precipitation).
Finally, students are prompted to reflect on their own learning process as they interrogate the quality of geographic information. The concepts of information gran ularity, accuracy, and provenance are examined in relation to each of the core content concepts. Using the concept of granularity, students understand that in some cases, they can refine their answers to previous questions when appropriate, but that overly specific answers can be less accurate. Students use the concept of provenance to interrogate their own understanding of how they derive answers from processed information and whether they can trust those answers. For more background information on the core concepts of spatial information, including definitions used in our teaching program, see Kuhn and Ballatore (2015).

\section{Designing a concept-based GIS course}

Concept-based instruction defines the foundational language that learners must acquire (Erickson, 2007). In this view, the central challenge of teaching GIS is articulating an appropriate set of concepts for GIS learners to acquire (Srivastava and Tait, 2012). Problem-based learning (PBL) is a complementary approach, which balances theory and application in designing activities to teach GIS concepts. With PBL, GIS learners actively apply concepts to solve real-world problems, often in consultation with a client (Keßler et al., 2018).

We structure an introductory GIS course around core concepts of spatial information (Kuhn, 2012) by focusing learning activities on software-independent foundational concepts. The course activities motivate students to use core concepts of spatial information to solve realistic problems; students are prompted to ask and answer questions about the real world in ways that underlie GIS software but are established independently of it.

The course was first designed in the fall of 2014 and has been updated every year until 2019. It is the first part of a series introducing undergraduate university students to GIS at UCSB. Most students pursuing a geography degree take this course, while those looking to become geographic information scientists take the yearlong series, which concludes with a capstone project addressing a real-world problem. Previous versions of the course offered a pragmatic approach to learning the history and techniques of GIS. Students were taught how to employ methods on generic types of geographic data. They learned a set of analytical techniques that would help them operate GIS software at a more advanced level than their untrained peers. Labs provided step-by-step instructions exclusively with ArcGIS.

The general principle we followed was to teach GIS by asking questions about spatio-temporal concepts, rather than the reverse (i.e., hoping to arrive at sensible questions by teaching software commands). A running example frequently mentioned in class involved the as- 
sessment of local solar energy potential with the goal of planning solar panel installations. Case studies showed how each concept was represented in data models and handled through typical operations on data in a GIS.

The weekly lectures related each core concept of spatial information to student experiences, discussing the views students held previously and showing possible shortcomings or misconceptions. Students submitted weekly questions in lectures, highlighting conceptual gaps in understanding. Refinements were made to the companion text for the course based on students' questions, which illustrated important ways of thinking that challenged the research.

\subsection{Software and data availability}

A description of the course version offered in 2017-including activities, lab materials, and data-is available online (https://github.com/saralafia/geog-gis-176). The lab assignments involved many tools, including web services, mobile apps, and online games. The lab tasks exemplified key questions around the core concepts without requiring students to engage much with software. The assignments encouraged students to explore and solve several problems around the concept as it occurs in a typical application area. The location, field, object, and event labs all required students to ask and answer questions about the university campus. For the field and object labs, the teaching assistants worked with the university library to curate a digital elevation model and building footprints of university buildings for students to use. In the network lab, the teaching assistants created a custom network dataset for the university campus from OpenStreetMap that included pedestrian walkways and bicycle trails. The event lab used data from campus administration on connections made to internet routers; students were excited to work with this event data when they realized it could serve as proxy for the number of people on campus at any given time. Each of the content labs added a conceptual layer to the students' GIS so they could understand how concepts could be used together; for example, students joined the internet connectivity data to the building footprints so they could visualize busy places on campus (e.g., large lecture halls during final exams). For the quality concept labs, students revisited data from earlier assignments to examine its granularity and accuracy, reinforcing the idea that the core concepts build upon each other.

\subsection{Course delivery}

One week of lectures and labs was devoted to each of the core concepts, spanning a quarter of instruction. In a semester system, allotting two weeks to each concept would be an attractive option, allowing for more discussions of applications, modeling approaches, and GIS projects. The weekly lectures followed a similar pattern for each concept. The course was deliberately sequenced to follow the order in which the concepts are presented in Section 3 beginning with the idea of location information, introducing the content concepts of field, object, network, and event, and concluding with the information quality concepts of granularity, accuracy, and provenance. The first lecture of each week related the core concept to past experiences of students, discussing the views students held previously, showing possible shortcomings or misconceptions, and illustrating the concept. Between the class meetings, students read a concise text on the concept and were encouraged to post questions of understanding in an online course forum, which were then discussed in the second lecture. The second lecture showed how GIS handle the concept in terms of data models and typical operations on the data, as well as applications. The examples focused mainly on the university campus, an experience shared by all students.

\subsection{Illustration of a concept-based lab}

Rather than guiding students through workflows, the labs encouraged students to ask and answer questions with a GIS. Students were given the following prompt: "Campus administration is interested in finding optimal locations for installing solar panels. Using your previous knowledge of location and fields and your new knowledge of objects, determine the best rooftops for installation." The data that students were given included a digital elevation model of terrain and a layer of university building footprints. Below are examples of questions from the activity and responses given by students, updated to reflect standard terminology:

- What is the location of a rooftop that might be suitable for solar panel installation? Use a spatial relation that holds between the rooftop and its surroundings (Figure 1).

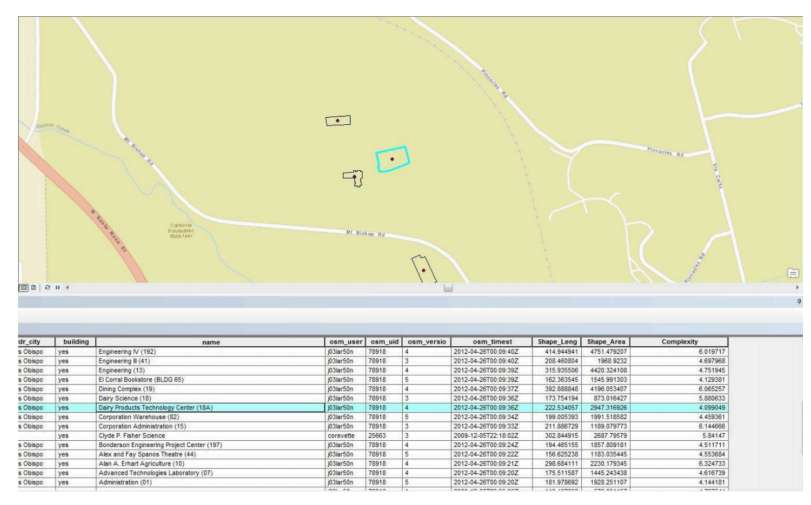

Figure 1. "The Dairy Products Technology Center (highlighted) is southeast of the building with Object ID 445. It's located between Pinnacles Rd. and the railroad tracks."

- Why aren't rooftops with varying heights optimal for solar panel installation? Remember that eleva- 
tion is often understood as a field, which is most commonly represented as raster data (Figure 2).

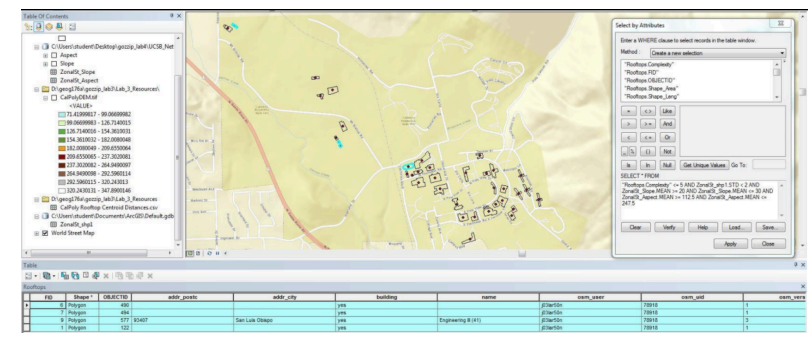

Figure 2. "I selected only those rooftops with a suitable shape, elevation values, slope, and aspect. I translated these criteria into a query for buildings with these attributes."

- Which buildings on campus would be suitable for solar panel installation based on all of the variables you have investigated? Remember that buildings are modeled as objects with attributes that we can query (Figure 3).

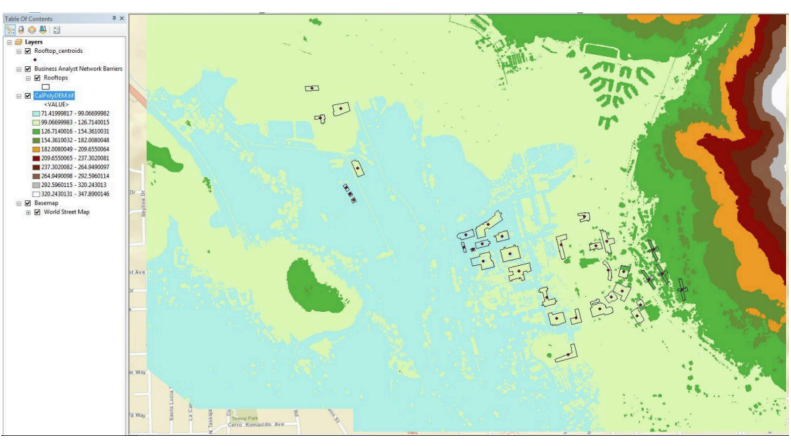

Figure 3. "I used the DEM to see which buildings have consistent heights because rooftops with different surface heights aren't optimal for solar panel installation."

Course activities guided students toward a high-level understanding of spatial computing, independent of software commands, but immediately applicable in the labs thanks to the focus on questions organized by core concepts and answered through GIS commands. Thus, students learn about spatial analyses through conceptualizations of geographic environments before they discover the GIS commands and data models required by the GIS to perform the analyses. This sequence appears more desirable for an introductory GIS course than starting with the organization of the commands in a particular GIS. The core concepts of spatial information offer learners a language for asking questions about the world and answering them using any GIS.

\section{Student impressions of the course}

At the conclusion of an early version of the course in 2016, we administered a survey to gauge student impressions; 66 of the enrolled 72 students participated in the survey. When asked what they liked most about lab activities, more than half of the students cited software training as a benefit despite our deliberate attempt to diminish the emphasis on use of software and emphasize problem-solving as a means of teaching GIS. Students also saw value in applying concepts to practical problems as part of the lab assignments, suggesting that activities succeeded in relating concepts to practical GIS analyses.

Many students felt that "the pace of the labs was too fast" and that "too much content was covered". This may be attributed to students learning how to use software and trying to solve an application problem at the same time. Some also remarked that switching tools and software between labs was challenging because they did not have previous experience and felt that they were expected to "pick everything up right away". Other complaints included the "lack of written and detailed instructions" in the labs. We expected that students would be willing to apply the software to problem-solving, as they would in an experimental laboratory course, yet, students still requested step-by-step guidance.

The majority of students $(68 \%)$ favored the network applications, suggesting that they may be the most intuitive to conceptualize. Student explanations included "I feel like the network section can be applied to a lot of scenarios... I like seeing how things are connected and those connections are measured", as well as "I liked it. . . because [the lab] was relatable and the instructions were clear'. Students' second favorite concept application area was events $(16 \%)$ as they were interested in dynamic mapping.

Following this survey, we redesigned all the labs to facilitate mapping questions to software commands. We reduced the workload per lab and the pace of instruction. Despite students' stated preference for learning software, instructional time remained focused on understanding a problem before learning how to navigate software menus.

Informal assessment strategies, such as ungraded quizzes, have occasionally been used in class to help students anticipate and address their conceptual gaps as the course progresses rather than relying on a single survey at the conclusion of the course. These assessments need to become much more frequent in order to provide more insights on how concept-based GIS teaching enables learners to transfer knowledge to application problems and other software products.

\section{Conclusion}

Our goal was to address limitations that students face when learning GIS from a traditional software command perspective, such as the long-term retention of methodological knowledge and the transfer of technical 
skills. We proposed an alternative teaching strategy to mitigate these challenges for students by allowing them to learn GIS from an information content perspective. We tested and refined this approach in several iterations of an undergraduate-level introductory GIS course. We then evaluated our approach by conducting a student survey and reflecting on lessons learned from a teaching perspective.

Our experience suggests that students can start learning GIS from a content, as opposed to software command, perspective. Most core concepts turned out to be relatively easy for new learners to acquire since they relate directly to daily experiences in situations like wayfinding, interpreting weather reports or using social networking tools. A rigorous focus on the core questions that a GIS helps answer encourages an understanding of GIS at a level above software menus, although this understanding is not what many students expect to gain in an introductory course. The main difficulty with designing labs was to develop unambiguous yet challenging prompts; questions that were too specific did not provoke enough critical thought and questions that were too vague inhibited students from reaching a common understanding. Students also struggled to grasp the ways that core concepts interact and guide applications, indicating opportunities to structure future versions of the course around a few in-depth case studies. We found that illustrating concepts through relevant applications engaged students with diverse interests.

The main obstacle to student satisfaction with the course was their expectation of learning a particular software package. Prioritizing question asking over button pushing challenged students to experiment and discover-something that many did not expect. We underestimated the difficulties associated with designing and guiding students through "hands-on" assignments that encouraged critical spatial thinking rather than recipedriven exercises for software commands. With "big spatio-temporal data" and "data science" entering so many human endeavors, a focus on information content rather than software commands appears more justified than ever, but remains difficult to convey. Calls to develop students' "critical spatial thinking" are also emphasizing the conceptual value of GIS education rather than positioning it simply as tool training (Bearman et al., 2016).

A UCGIS survey of instructors who adapted their courses for remote learning between September and December 2020 highlighted opportunities for innovation in GIS education (Bowlick and Shook, 2020). The survey found that teaching a primarily software-based curriculum remotely was challenging; instead, instructors that switched to web- and open-source GIS tools, combined with flipped classroom assignments where students worked collaboratively during class meetings, saw greater satisfaction.
We also anticipate other trends, such as the inclusion of spatial sciences in emerging data science curriculum. Efforts to incorporate GIS into the data science curriculum also require a vocabulary of spatial computing that is clear and powerful (Rey et al., 2020). We believe that providing interdisciplinary learners with core concepts that support spatial questions is a first step toward expanding access to GIS-like functionality in any form.

As research is solidifying the core concepts, the literature and didactic take-up are gradually improving and a book addressing life-long learning needs of professionals as well as graduate students is in preparation. In a future form of practice, the core concepts are envisioned to serve as bridges between analysts' questions or hypotheses and the rapidly growing variety of GIS models, tools, and workflows (Scheider et al., 2017). The core concepts have also been used to evaluate students' spatial thinking, for example offering a taxonomy to describe features observed when interpreting thematic maps (Ishikawa, 2016). At a more general didactic level, the core concepts offer constructive alignment by segmenting course content into discrete stages (Etherington, 2016). Our teaching and research efforts continue to pursue core concepts of spatial information that support spatial questions and answers through GIS queries and workflows.

Acknowledgements. This work was supported by UCSB's Center for Spatial Studies. Thomas Hervey, Behzad Vahedi, Jingyi Xiao, Rafael Ramos, and Marcela Suárez (UCSB Geography) contributed to the development of course materials and Crystal Bae (UCSB Geography) provided valuable review and editorial feedback. Mindy Colin and Lisa Berry (UCSB Center for Innovative Teaching, Research, and Learning) provided inspiring consultations and workshops on pedagogy that supported the redesign of the course.

\section{References}

Albrecht, J.: Universal analytical GIS operations: A taskoriented systematization of data structure-independent GIS functionality, Geographic Information Research: Transatlantic Perspectives, pp. 577-591, 1998.

Bearman, N., Jones, N., André, I., Cachinho, H. A., and DeMers, M.: The future role of GIS education in creating critical spatial thinkers, Journal of Geography in Higher Education, 40, 394-408, 2016.

Bolstad, P.: GIS Fundamentals: A First Text on Geographic Information Systems, Eider Press, 2005.

Bowlick, F. and Shook, E.: UCGIS Quick Education Survey Summary: September-December 2020, Tech. rep., UCGIS, https://www.ucgis.org/assets/docs/Committees/ Education/UCGIS, 2020.

Camara, G., Egenhofer, M. J., Ferreira, K., Andrade, P., Queiroz, G., Sanchez, A., Jones, J., and Vinhas, L.: Fields as a generic data type for big spatial data, in: International 
Conference on Geographic Information Science, pp. 159-172, Springer, 2014

Couclelis, H.: Ontologies of geographic information, International Journal of Geographical Information Science, 24, 1785-1809, 2010.

DeMers, M. N.: Using intended learning objectives to assess curriculum materials: The UCGIS body of knowledge, Journal of Geography in Higher Education, 33, S70-S77, 2009.

Erickson, H. L.: Stirring the head, heart, and soul: Redefining curriculum, instruction, and concept-based learning, Corwin Press, 2007.

Etherington, T. R.: Teaching introductory GIS programming to geographers using an open source Python approach, Journal of Geography in Higher Education, 40, 117-130, 2016.

Gao, S. and Goodchild, M. F.: Asking spatial questions to identify GIS functionality, in: 2013 Fourth International Conference on Computing for Geospatial Research and Application, pp. 106-110, IEEE, 2013.

Goodchild, M. F.: Geographical information science, International journal of geographical information systems, 6, 31-45, 1992.

Goodchild, M. F.: The fourth R? Rethinking GIS education, ESRI ArcNews, 28, 1, 2006.

Goodchild, M. F., Yuan, M., and Cova, T. J.: Towards a general theory of geographic representation in GIS, International Journal of Geographical Information Science, 21, 239-260, 2007.

Howarth, J. T. and Sinton, D.: Sequencing spatial concepts in problem-based GIS instruction, Procedia-Social and Behavioral Sciences, 21, 253-259, 2011.

Ishikawa, T.: Spatial thinking in geographic information science: Students' geospatial conceptions, map-based reasoning, and spatial visualization ability, Annals of the American Association of Geographers, 106, 76-95, 2016.

Kemp, K. K. and Goodchild, M. F.: Developing a curriculum in geographic information systems: The National Center for Geographic Information and Analysis core curriculum project, Journal of Geography in Higher Education, 15, 123 134, 1991.

Kemp, K. K., Goodchild, M. F., and Dodson, R. F.: Teaching GIS in geography, The Professional Geographer, 44, 181-191, 1992.

Kerski, J. J., Demirci, A., and Milson, A. J.: The global landscape of GIS in secondary education, Journal of Geography, 112, 232-247, 2013.

Keßler, C., Hansen, H. S., Schrøder, L., and Arsanjani, J. J.: Teaching Geospatial Technologies with Problem-Based Learning, in: Teaching Geospatial Technologies to All, 2018.

Kidman, G. and Palmer, G.: GIS: The technology is there but the teaching is yet to catch up, International Research in Geographical and Environmental Education, 15, 289-296, 2006.

Kuhn, W.: Core concepts of spatial information for transdisciplinary research, International Journal of Geographical Information Science, 26, 2267-2276, 2012.

Kuhn, W. and Ballatore, A.: Designing a language for spatial computing, in: AGILE 2015, pp. 309-326, Springer, 2015.
Land, R., Meyer, J. H., and Flanagan, M. T.: Threshold concepts in practice, Springer, 2016.

Longley, P. A., Goodchild, M. F., Maguire, D. J., and Rhind, D. W.: Geographic information science and systems, John Wiley \& Sons, 2015.

Raper, J. and Green, N.: Teaching the principles of GIS: lessons from the GISTutor project, International Journal of Geographical Information Systems, 6, 279-290, 1992.

Rey, S. J., Arribas-Bel, D., and Wolf, L. J.: Geographic Data Science with Python, CRC Press, 2020.

Rickles, P., Ellul, C., and Haklay, M.: A suggested framework and guidelines for learning GIS in interdisciplinary research, Geo: Geography and Environment, 4, e00 046, 2017.

Scheider, S., Ostermann, F. O., and Adams, B.: Why good data analysts need to be critical synthesists. Determining the role of semantics in data analysis, Future Generation Computer Systems, 72, 11-22, 2017.

Schulze, U.: "GIS works!"-But why, how, and for whom? Findings from a systematic review, Transactions in GIS, 2020.

Srivastava, S. K. and Tait, C.: An activity-based learning approach for key geographical information systems (GIS) concepts, Journal of Geography in Higher Education, 36, 527545, 2012.

Talmy, L.: How language structures space, in: Spatial orientation, pp. 225-282, Springer, 1983.

Vahedi, B., Kuhn, W., and Ballatore, A.: Question-based spatial computing - a case study, in: Geospatial Data in a Changing World, pp. 37-50, Springer, 2016.

Wikle, T. A. and Fagin, T. D.: GIS course planning: A comparison of syllabi at US college and universities, Transactions in GIS, 18, 574-585, 2014.

Wright, D. J., Goodchild, M. F., and Proctor, J. D.: GIS: tool or science? Demystifying the persistent ambiguity of GIS as" Tool" versus" Science", Annals of the Association of American Geographers, pp. 346-362, 1997.

Zhu, R., Kyriakidis, P. C., and Janowicz, K.: Beyond pairs: generalizing the geo-dipole for quantifying spatial patterns in geographic fields, in: The Annual International Conference on Geographic Information Science, pp. 331-348, Springer, 2017. 